Guest Editorial

\title{
Poverty and Malnutrition
}

\author{
${ }^{1}$ Syed Abdul Wasay Tanweer Hameed* \\ ${ }^{1}$ Faimah Jinnah College of Medicine \& Dentistry
}

How to Cite:

Tanweer Hameed, S. A. W. (2020). Poverty and

Malnutrition: Poverty and

Malnutrition. DIET FACTOR

(Journal of Nutritional \&Amp;

Food Sciences), 1(02).

https://doi.org/10.54393/df.v1i0

2.8

Corresponding author:

Syed Abdul Wasay Tanweer

Hameed

Faimah Jinnah College of

Medicine \& Dentistry, Lahore,

Pakistan

wasaytanweer25@gmail.com

\section{Article History}

Received: $23^{\text {rd }}$ July 2020

Accepted: $28^{\text {th }}$ August 2020

Published: $30^{\text {th }}$ December 2020
A diet is considered balanced when it contains adequate amounts of carbohydrates, proteins, fats, vitamins, minerals, fiber and water. A well balanced diet fulfills an individual's daily metabolic requirements. In order to live a healthy life one must eat a balanced diet at all times, and to eat a balanced diet one should incorporate a variety of foods in one's daily routine. Complications arise when these daily nutritional requirements are not met, and over a prolonged period of time certain diseases arise as a result of absolute deficiency or excess of one or more essential nutrients.

Malnutrition is a common condition caused by poorly balanced diet or a diet that is unable to meet an individual's daily nutritional needs. It often manifests in children and in povertystricken areas. The major forms of malnutrition are undernutrition, overnutrition, imbalance and specific deficiency. According to WHO guidelines, a balanced diet must contain sixty percent of carbohydrates, twenty percent of proteins, ten percent of fats and remaining percentage of vitamins and minerals. The sources from which one can obtain certain nutrients solely depend on one's socioeconomic background status. Poverty is the main reason behind malnutrition in Pakistan, especially in the rural areas of Sindh and Punjab. The factors that lead towards malnutrition are closely intertwined with geopolitical, social and economical conditions of an individual.

According to National Nutrition Survey Pakistan, thirty-one percent of children are underweight, forty-three percent have stunted growth, fifteen percent show wasting, fifty percent are anemic and almost all of them live in poverty. Complications like stunted growth, mental retardation and poor health in children are common. The data also shows that the young children are most vulnerable to malnutrition and majority of children under the age of five are severely affected.

Chronic diseases like kwashiorkor, marasmus, anemia and several vitamin deficiencies develop at an early stage of life. Inadequate food intake, poor food habits and scarcity of food leads towards such dire conditions of children in rural areas of Pakistan. Socioeconomic status of these children hinders their growth as their families are unable to provide them with proper nutrition. Their bodies require proper nutrition and diet to grow. Disproportionate diet and deficiency of certain nutrients result in a variety of complications and diseases. Deficiency of vitamin A can cause eye problems, similarly deficiency of vitamin B complex can result in impaired mental health development, weakness and anemia. Vitamin C deficiency causes scurvy and vitamin D deficient diet can cause rickets in children. Several minerals like calcium, iron, sodium, iodine and magnesium are also vital for one's life. Deficiencies of such vital minerals can result in tetany, goiter and several other problems as well.

Poor socioeconomic background and ever increasing inflation rate of the country makes it impossible for an impoverished family to fulfill it's basic nutritional requirement. More than sixty percent of rural population live below poverty line in Pakistan. They are unable to afford and buy meat or fruits on regular basis. Their staple diet consists mainly of carbohydrates and some fats. Meat, fruits and some vegetables are too expensive for an individual to buy and almost out of their reach. From the very beginning a child is deprived of essential micronutrients and macronutrients unintentionally. Poor maternal health also contributes towards the problem. Mothers who are malnourished at the time of their child's birth, can experience 
DOI: https://doi.org/10.54393/df.v1i02.8

several complications. It can result in abortion, premature birth, low birth weight of the baby or the baby could face problems such as mental retardation and developmental difficulties. Malnourished mothers are also unable to breastfeed their children without risking their own nutritional status. Lack of knowledge and lack of resources also contribute towards such pathological conditions.

Half of the problem would be solved if one knew the importance of nutrition in one's daily life. A proper diet should include all the essential nutrients. If meat or fruits are out of reach, one can resort to less expensive but fulfilling alternates. Protein's main sources are meat, poultry and dairy products. Nonetheless, proteins can also be obtained from legumes, grains and several green vegetables, which are less expensive and easily available. Health education and information can be provided to people that will allow them to make healthy choices. Government should also play it's part and help them reach their goal, and eradicate malnutrition from the country. 